\title{
Cyberbikes - Interactive Visualization of Manufacturing Processes in a Virtual Environment
}

\author{
Prof. Dr.-Ing. J. Gausemeier \\ Dipl.-Ing. O. von Bohuszewicz \\ Dr.-Ing. P. Ebbesmeyer \\ Dipl.-Ing. M. Grafe
}

Heinz Nixdorf Institut

Universität-GH Paderborn

Fürstenallee 11, 33102 Paderborn

Germany

Tel.: +49 $525160-6267$

Fax.: +495251 60-6268

grafe@hni.uni-paderborn.de

\begin{abstract}
This paper describes CYBERBIKES, a real-time simulation of an enterprise that produces bicycles. The system is designed as a virtual environment. It is based on a realistic 3-D model of an industrial enterprise coupled with the representation and control of production processes. Furthermore, the flow of information like production data is visualized by means of clear metaphors. The user is immersed in the virtual environment by using a head mounted display through which he/she can explore the various departments of the industrial enterprise and learn how information technology influences modern production processes.
\end{abstract}




\section{INTRODUCTION}

\section{Statement of purpose}

A modern industrial enterprise is a very complex system. The current marketplace requires flexibility and a high rate of productivity. To meet these challenges business managers have to reengineer and optimize their business processes by making extensive use of information technology (IT) and sophisticated production technology.

Because of its complexity, it is often difficult to understand how a modern industrial enterprise really works. Specifically, how do the various different business processes interact with each other? How do they contribute to the results? How can IT contribute to increase productivity? A thorough understanding is crucial to those who operate it. It is even more important for those who will operate such an enterprise in the future - namely the students of industrial engineering.

One obvious solution to the foregoing question is to built one just for purpose of study. In Germany, for example, a series of model factories have been set up in recent years [Wal93]. These CIM-Centers (Computer Integrated ManufacturingCenters) consist of real systems such as manufacturing systems for the production of parts, assembly systems and automated transport systems. The major drawback of building model factories for research, educational and training purposes is the high cost of the set-up, operation and maintenance of these facilities.

Simulators have successfully been used to teach students about complex systems. Flight simulators are well-known examples for these types of training devices. Today, Virtual Reality is a very useful technology to build simulators for a wide variety of purposes. This paper describes a system called CYBERBIKES which immerses a person in a virtual environment which simulates an industrial enterprise (Figure 1). The main purpose of the system is to teach students of mechanical and industrial engineering how a complex industrial enterprise works.

\section{Objectives}

The basic idea of CYBERBIKES is to supply students with a system which allows them to explore a modern industrial enterprise. An appropriate system should give the user the impression of "really being there“. The clearest solution to this requirement is to design the core of the system as a Virtual Environment based on realistic looking 3-D models representing all objects a typical industrial enterprise consists of - i.e., the plant building, the various departments of the production plant with its machine tools, robots and transportation systems, the offices of engineering, administration and management, and perhaps even a cafeteria. 


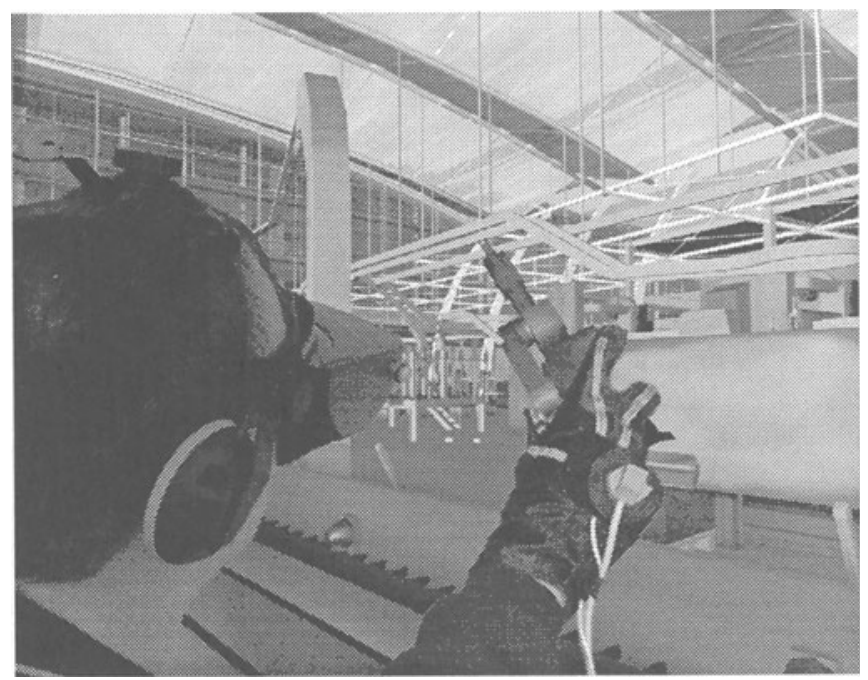

Figure 1: A user immersed in the CYBERBIKES virtual Environment

Although a realistic looking 3-D model is the most important part of such an virtual environment there are other issues which have to be addressed. A simulation of an industrial enterprise must also represent the data to be processed within the production. For example machine tools and robots have to operate like they do in reality. Thus, there is a need for special simulation models.

The challenge is to implement CYBERBIKES as a virtual environment. Graphics rendering of complex 3-D models, 3-D sound, simulation of machine tools and robots, clear simulation of material flow and the flow of information have to operate in real-time. Furthermore, there is a need to integrate software which enables a person to control the various business processes. The most realistic solution would be to use real IT applications -- i.e., software which is used in the actual world of an industrial enterprise -- e.g. manufacturing control systems (MCS). Real IT applications should be integrated whenever considered reasonable. At the same time simplifications often are unavoidable for reasons of feasibility.

\section{GENERAL APPROACH}

CYBERBIKES is a virtual environment representing a medium-sized enterprise which produces high-quality bicycles. The bike was chosen because it is a wellknown product to everybody, though the idea behind the system is not limited to bicycles.

CYBERBIKES was designed from the scratch starting with the basic design of the production processes. These are based on actual production processes which can be 
found in modern bike manufacturing industry. The basic design has been reworked in several different forms resulting in a detailed design. At this stage the space requirements for the plant building were defined as well as how many and what types of machine tools and production systems were needed. At the end of the detailed design stage all the information necessary to start geometric and nongeometric modeling were available (see section 3 ).

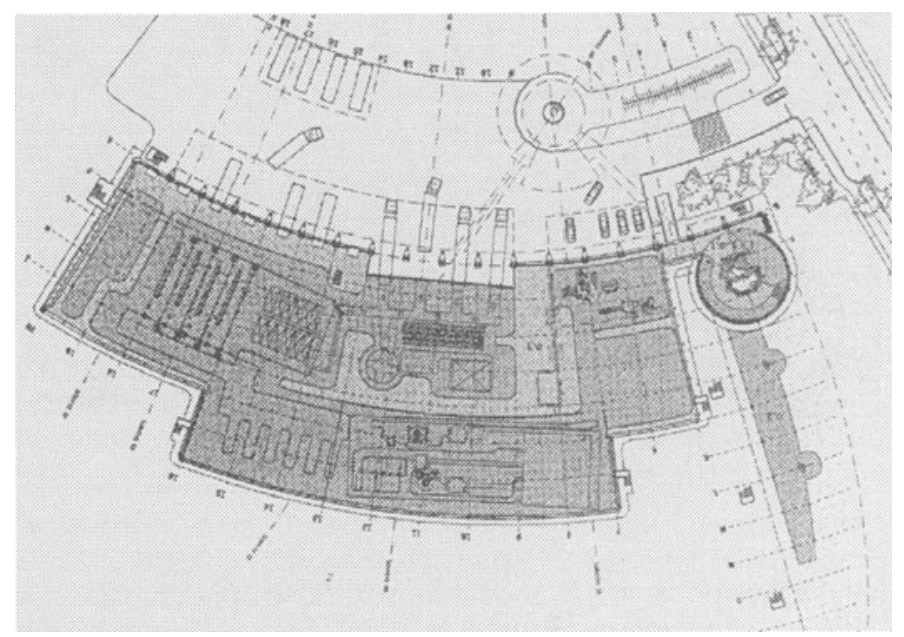

Figure 2: CYBERBIKES floor plan

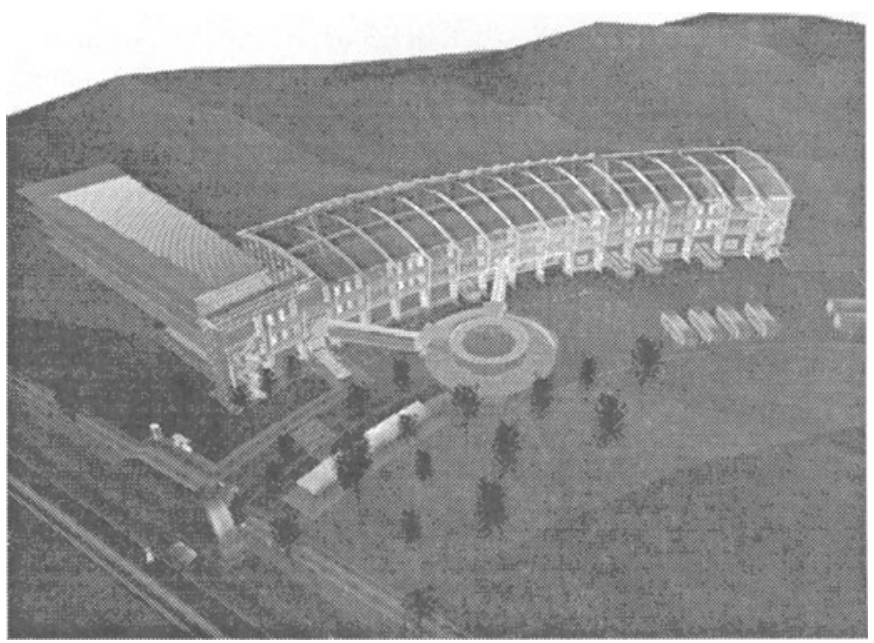

Figure 3: Bird's-eye view of CYBERBIKES 


\section{System Overview}

To explore the CYBERBIKES virtual environment the user puts on a head mounted display (HMD) enabling him/her to move through the model and interact with objects within the virtual environment using a special kind of 3-D input device called a ,3-D-mouse“. During the walkthrough of the scene, standard magnetic tracking devices track the user's head and hand. The virtual environment itself is generated and controlled by a four processor graphics workstation (SGI Onyx). The workstation is equipped with two separate graphics subsystems to generate stereoscopic images. Acoustic rendering is carried out by an entry level workstation (SGI Indy) which drives an 4-speaker ambisonic audio subsystem [Bam90].

The system is installed in the "Software Theater", a cinema-like auditorium equipped with 30 seats and a large-scale stereoscopic projection screen (Figure 4 and Figure 5). This facility, located in the Heinz Nixdorf MuseumsForum next to our institute, was designed by our group for the presentation of CYBERBIKES and other virtual reality applications.

In the Software Theater trained instructors offer visitors guided tours to the CYBERBIKES virtual environment. The audience follows the instructors view presented through the HMD he wears on a large-scale projection screen. Interested users who wish to explore CYBERBIKES themselves can use the HMD while the instructor can lead them to points of interest.

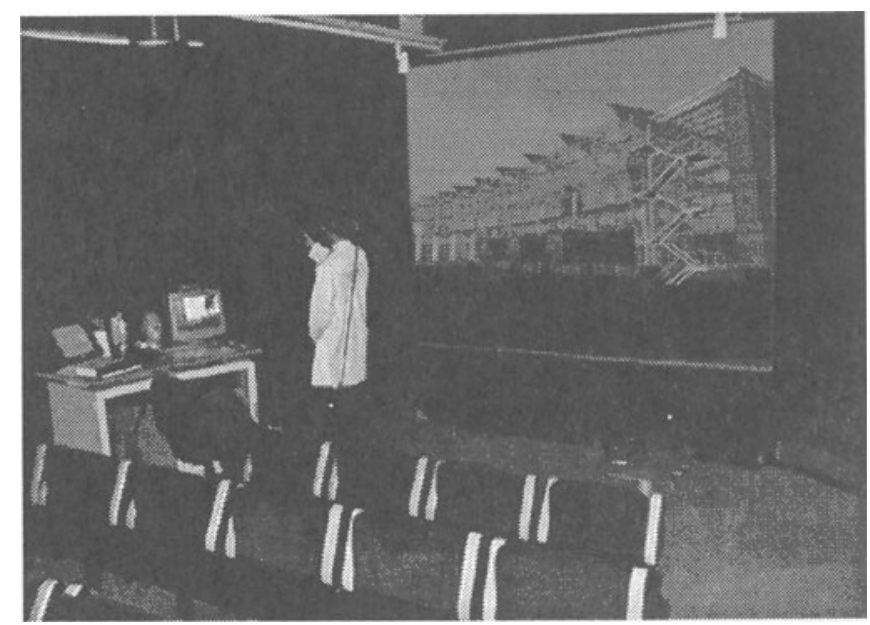

Figure 4: The Software Theater used for presentation of the Cyberbikes virtual environment 


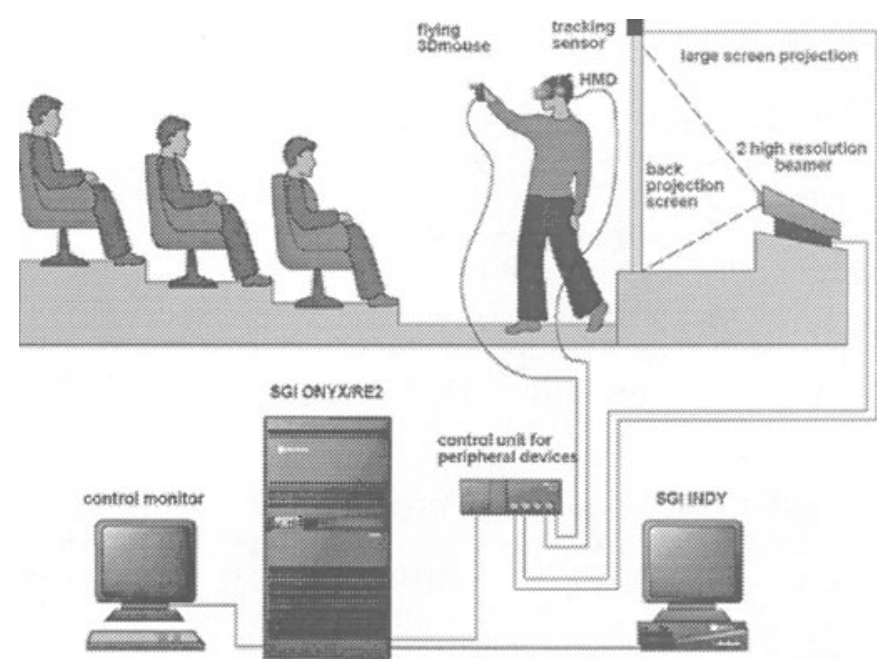

Figure 5: Schematic layout of the Software Theater

\section{Training Procedure}

After the user has entered the CYBERBIKES production, he is able to start a production process using a manufacturing control system (MCS) and watch the working numerical controlled machine tools and industrial robots. The results of his/her interaction are visualized by the manufacturing control system immediately. The user is also able to modify the production process currently running. The manufacturing control system (MCS) is an example of software that can be found in a real world enterprise. We have integrated such a system into CYBERBIKES [GBG+96]. The graphical user interface of the MCS is displayed on a virtual monitor. The user immersed in CYBERBIKES interacts with the system by means of a touch screen metaphor using hand, which is represented in the virtual environment by a 3-D hand icon.
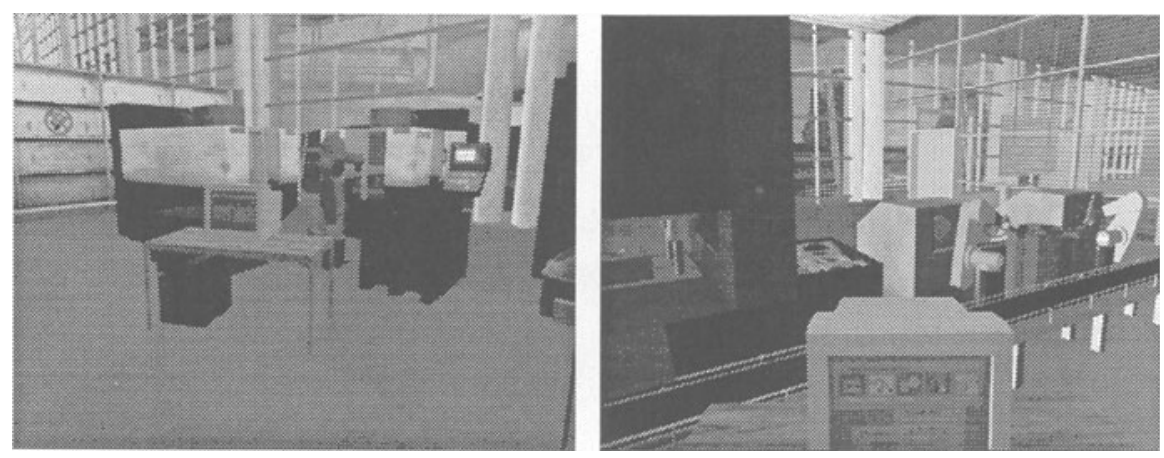

Figure 6: Terminals in the manufacturing area 


\section{SYSTEM DESIGN AND IMPLEMENTATION}

\section{Geometric Modeling}

All geometric modeling was done with 3-D CAD tools. The plant building itself and the interior of the offices were designed by the department of architecture at the university of fine arts in Berlin, Germany. All other models were created at our institute. The machine tools and industrial robots were modeled with the help of drawings and photographs of real machine tools used for bicycle production. Realism of the models was enhanced by using realistic materials and texture maps. Furthermore, a lot of time was spent optimizing the models for the use in a realtime environment. The whole geometric data set of CYBERBIKES consists of $1,357,000$ polygons. 129 megabytes are required to store it in a compact binary data format (108 MB geometry files and $21 \mathrm{MB}$ texture files). Figure 7 shows a selection of different inner and outer views from CYBERBIKES. They underline the complexity and the level of realism of the CYBERBIKES virtual environment.

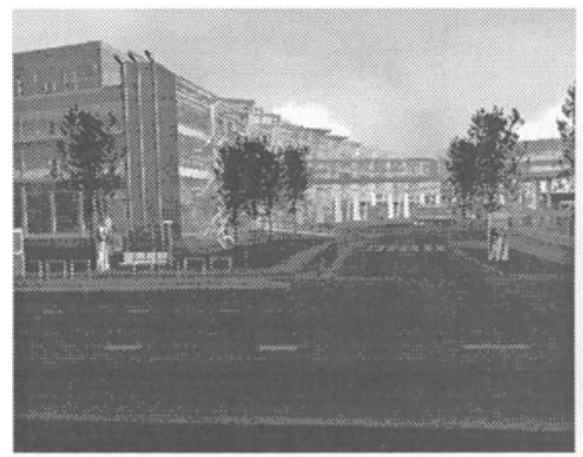

(a)

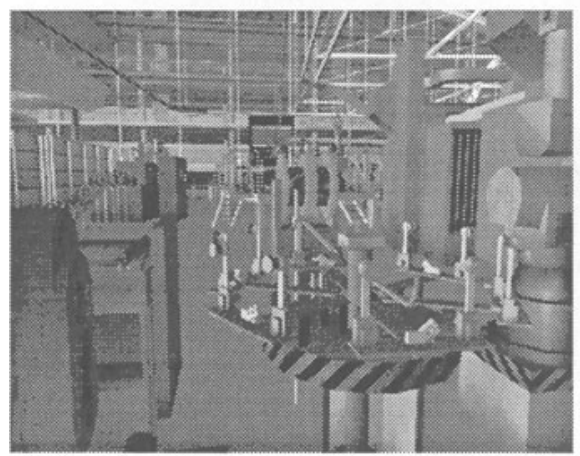

(c)

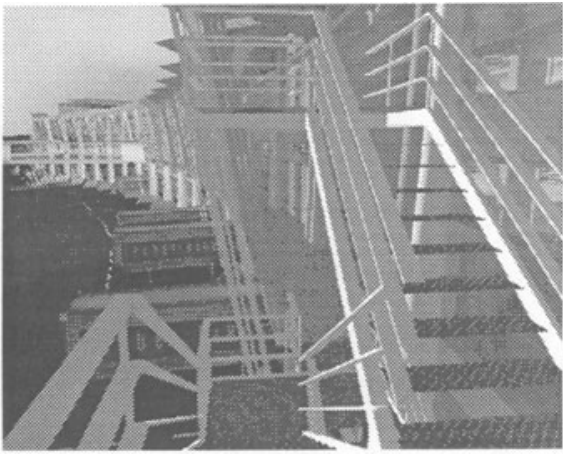

(b)

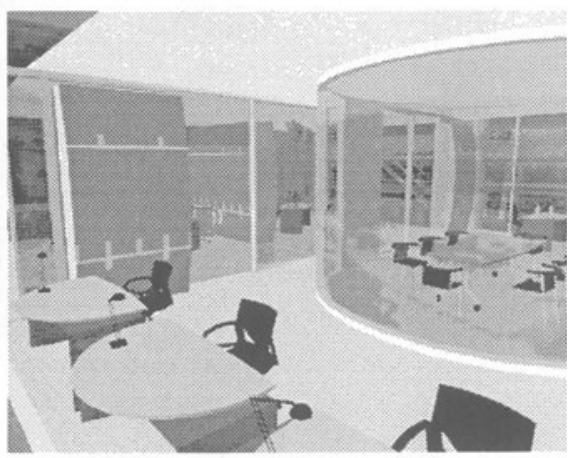

(d)

Figure 7: Views from the CYBERBIKES virtual environment 
Real industrial enterprises are operated by actual human labor. Thus, human beings should also be included in a realistic simulation of an industrial enterprise. Realistic models of human beings for the use in real-time environments are difficult to create [DM95], especially when numerous representations are needed simultaneously. In most existing VR-applications humans look and behave like toys. We decided to make light of this situation by specifically modeling humans as toys (Figure 8). We believe this is an acceptable solution for our application.

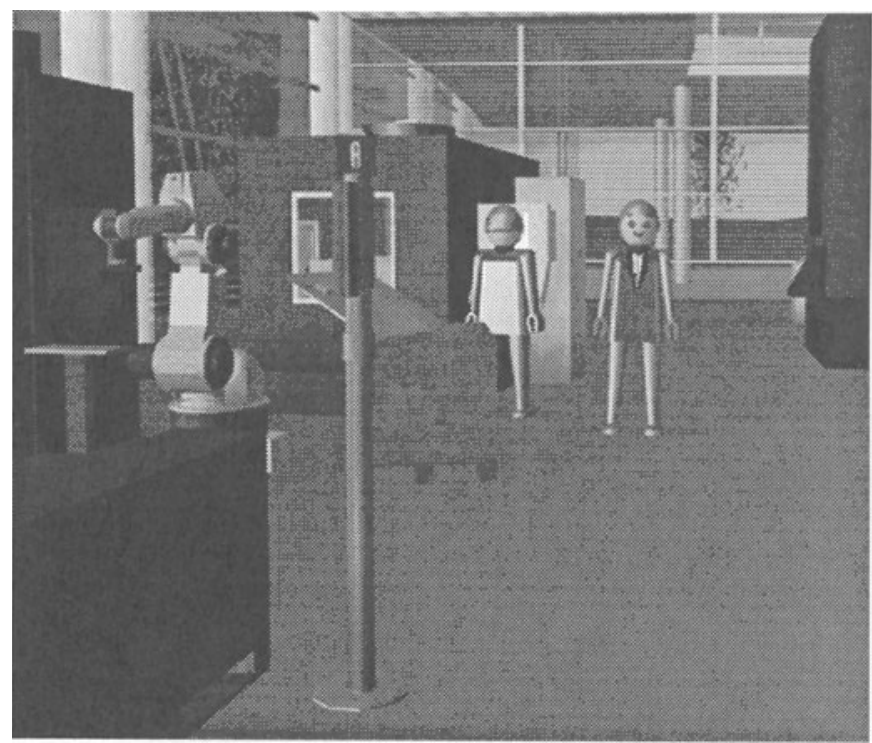

Figure 8: Toy models representing workers in the pedal crank production

\section{Non-Geometric Modeling}

Data-processing in modern industrial enterprises is hierarchically structured into four management layers, ranging from issuing production orders with declared delivery dates to control of particular machine components, and where each layer processes specific tasks.

Planning and Disposition Layer: The management of primary data and material management occurs at this layer. Customer orders are managed and handed on to the next layer.

Manufacturing Control Layer: At this layer, the customer orders received from the planning and disposition layer, are transformed into manufacturing orders. At this stage, planning of details is carried out -- i.e., staffing schedules of particular machines are generated by considering the predetermined delivery dates taken from customer orders. As a part of the manufacturing layer NC-programs are passed to machines on demand. In addition the manufacturing layer ensures the 
efficiency of the production run. Reports of disturbances are collected and forwarded to subsequent applications.

Process Control Layer: This layer is responsible for the coordination of the production equipment and the specific transport systems. Records of material flow in the production process are kept here and processing data of the individual machines is registered for quality control.

Machine Control Layer: This is the lowest management layer. It contains the controls for all automated manufacturing machines.

We have included the most important parts of each layer into the non-geometric model of the CYBERBIKES virtual environment. It is a simplified model of production control in an industrial enterprise. The user of CYBERBIKES is able to process customer orders. These orders generate manufacturing instructions which are subsequently forwarded to manufacturing units consisting of machine tools and industrial robots. The flow of information associated with these processes is invisible in the real world. In the CYBERBIKES virtual environment however, users can see and thus understand the flow of information. Information like data is visualized by means of metaphors as shown in Figure 9.

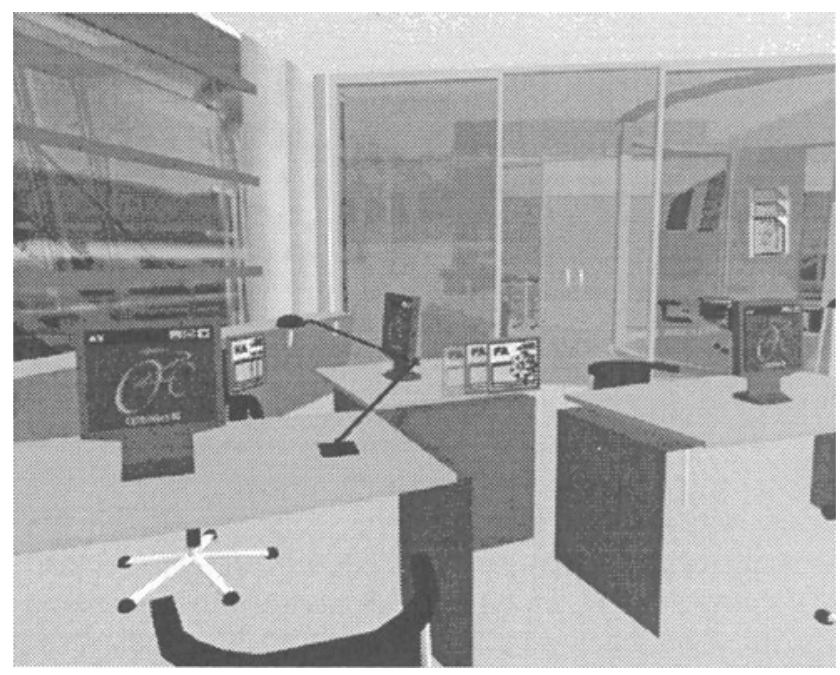

Figure 9: Visualization of the flow of information within CYBERBIKES.

(Data packages moving from one host to another)

\section{Real-Time Environment}

The simulation model for the CYBERBIKES virtual environment comprises all geometric and non-geometric models as shown in Figure 10. 


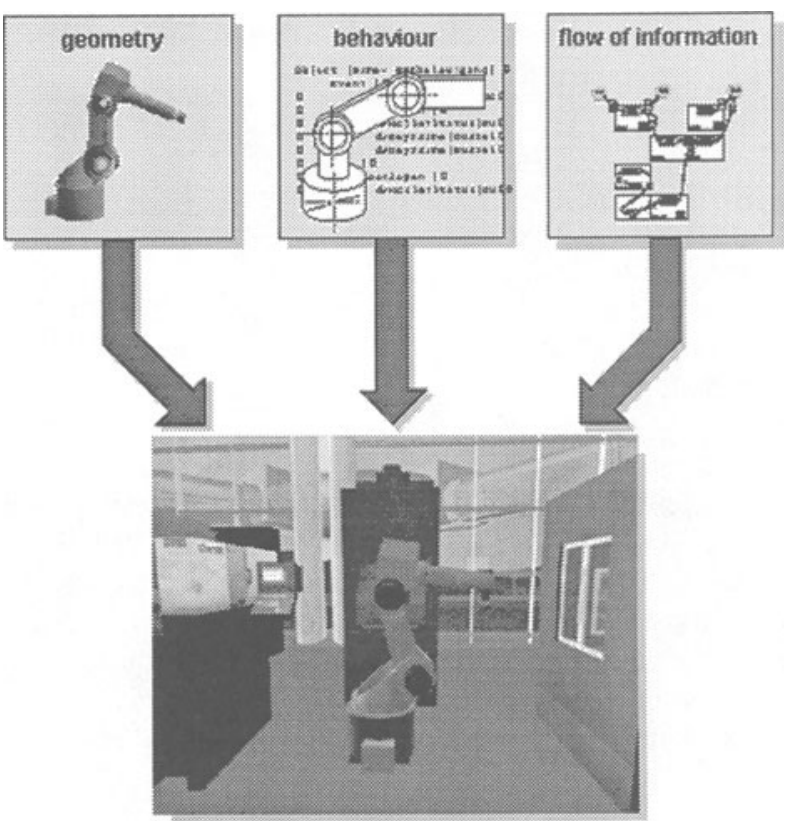

Figure 10: The parts of the simulation model

The simulation model is executed through the real-time environment. The most important requirement of the system is to deliver real-time frame rates (more than 15 frames/sec). This crucial to maintain the illusion of a interactive virtual world. The hardware resources needed to process the complete CYBERBIKES dataset in real-time is nearly a magnitude greater than what state of the art graphics hardware delivers today.

In recent years the increase in complexity of virtual environments has outpaced the improvements of graphics hardware. Thus there is an ongoing research in reducing the geometric complexity of a given scene for real-time rendering. Among others the most important strategies are level-of-detail hierarchies [Cla96], [MS95], visibility culling [GKM93], occlusion culling [ZMH+97] and image based rendering utilizing texture maps [Sch96], [Ebb97]. The CYBERBIKES virtual environment utilizes several of these advanced rendering techniques. The implementation is based on a special purpose API (Application Programming Interface) for real-time rendering [RH94].

\section{EVALUATION OF THE SYSTEM}

A first version of CYBERBIKES was completed in October 1996. This version consisted of the plant building and $20 \%$ of the production systems. At that time no 
offices were included. The virtual environment has been continuously updated since then. Several production systems as well as office areas have been added. CYBERBIKES is scheduled to be completed in December 1997.

Since the initial version of the system was available CYBERBIKES has been presented and tested in the Software Theater mentioned above. Since then more than 50,000 people have seen these presentations. $10 \%$ of the visitors used the HMD themselves. The majority of those who have immersed themselves in CYBERBIKES got a positive impression. There where a number of complaints about the low resolution of the HMD. Several users had difficulties finding their way around when being immersed in the CYBERBIKES virtual environment. It is obviously necessary to have efficient navigational aids in virtual environments of such complexity. A detailed study on the usability of the system to find out whether it meets its design goals will be done shortly after its completion.

\section{CONCLUSIONS}

We have described CYBERBIKES, a real-time simulation of a typical industrial enterprise, based on a 3-D virtual environment. The core of the system is a realistic 3-D model of an industrial plant. The user is able to explore the model of the virtual enterprise by means of immersing himself/herself in this environment using a head mounted display. Besides the geometric model the systems includes a model which represents the control of the production processes. Flows of information associated with these processes are visualized. The user gets the illusion of being in an industrial enterprise which is set apart from the real world by explaining itself through enhanced visualization techniques. Future work on the system will be focused on adding navigational aids. This could be accomplished by introducing agents and smart maps. A further area of research will include extending CYBERBIKES to a multi-user environment where more than one user can be immersed simultaneously.

\section{ACKNOWLEDGMENTS}

We would like to thank the Heinz Nixdorf MuseumsForum (www.hnf.de) and the Westfalen Foundation for supporting this work. Furthermore we would like to thank all the students involved in this project. 


\section{REFERENCES}

[Bam90] Bamford, Jeffrey S.: An Analysis of Amibisonic Sound Systems of First and Second Order; Master's Thesis; University of Waterloo, Waterloo, 1995

[Cla76] Clark, J. H.: Hierarchical Geometric Models for Visible Surface Algorithms Communications of the ACM, 19, 10 (October 1976)

[DM95] Durlach, N. I.; Mavor, A. S. (Editors): Virtual Reality: Sientific and Technological Challenges, National Research Concil, National Academy Press, Washington DC, 1995

[Ebb97] Ebbesmeyer, P.: Dynamische Texturwände - Ein Verfahren zur echtzeitorientierten Bildgenerierung für Virtuelle Umgebungen technischer Objekte, Dissertation, Heinz Nixdorf Institut, Universität-GH Paderborn, 1997

[GBG+96] Gausemeier, J.; von Bohuszewicz, O.; Gehnen, G., Grafe, M.: Cyberbikes: An Immersive Virtual Environment controlled by real CIMApplications. Proceedings of the FIVE 1996 Conference, Pisa, December 1996

[GKM93] Greene, N.; Kass, M.; Miller G.:Hierarchical Z-Buffer Visibility, Proceedings of SIGGRAPH '93, in ACM Computer Graphics, 1993

[MS95] Maciel, P. W. C.; Shirley, P.: Visual navigation of large environments using textures clusters, Proceedings 1995 Symposium on Interactive 3-D Graphics, June 1995

[RH94] Rohlf, J.; Helman, J.: IRIS Performer: A High Performance Multiprocessing Toolkit for Real-Time 3-D Graphics, Proceedings of SIGGRAPH '94, in ACM Computer Graphics, 1994

[Sch96] Schaufler, G.: Exploiting Frame-to-Frame Coherence in a Virtual Reality System, Proceedings of IEEE VRAIS ‘96, 1996

[Wa193]Walze, H. (ed): Computer Integrated Manufacturing (CIM) - Results from Projects within the Manufacturing Technologies Programme 1988 1992, Kernforschungszentrum Karlsruhe GmbH, PFT-Report, KfK/PFT 171, 1993

[ZMH+97] Zhang, H.; Manocha, D.; Hudso, T.; Hoff, K. E.: Visibility Culling using Hierachical Occlusion Maps, Proceedings of SIGGRAPH '97, in ACM Computer Graphics, 1997 